\title{
The Prevalence of Ectopic Gestation: A Five-Year Study of 1273 Cases
}

\author{
Yan-Yan Fan' \\ Yi-Nan Liu ${ }^{2}$ \\ Xin-Tong $\mathrm{MaO}^{3}$ \\ Yan Fu' \\ 'Department of Obstetrics and \\ Gynecology, The First Hospital of Jilin \\ University, Changchun, I3002I, People's \\ Republic of China; ${ }^{2}$ Department of \\ Otorhinolaryngology, Jilin Province Faw \\ General Hospital, Changchun, I300II, \\ People's Republic of China; ${ }^{3}$ Department \\ of Obstetrics and Gynecology, Beijing \\ Hospital of Traditional Chinese Medicine, \\ Beijing, I0010, People's Republic of China
}

Correspondence: Yan-Yan Fan

Tel +8643188785228

Fax +8643188785228

Email fanyanyan@jlu.edu.cn
Objective: This study aims to investigate the prevalence, related risk factors, manifestations, and management of ectopic pregnancy in the first hospital of the Jilin University over a five-year period.

Methods: A retrospective study of ectopic pregnancy was conducted in the First Hospital of the Jilin University between January 1, 2010 and December 31, 2014. The results were analyzed using simple descriptive statistics and reported as frequencies and percentages.

Results: The results revealed that out of 16,050 gynecological admissions to the hospital over the five-year period, there were 1273 ectopic pregnancies, with a prevalence rate of $7.93 \%$ of all gynecological admissions. The majority of these patients were aged 25-34 y and had a past history of abortion (61\%) and uterine cavity surgery $(38.6 \%)$, and a significant number were nulliparous $(549,43.1 \%)$. Bleeding accompanied by abdominal pain were the most common presenting complaints $(65.2 \%)$. A unilateral salpingectomy was performed for most of these patients.

Conclusion: Ectopic pregnancy had notable morbidity over the five-year period under study, and a history of abortion and uterine cavity surgery were identified as associated risk factors that limited the future reproductive potential of nulliparous women. Therefore, targeted health education campaigns should be conducted in order to enlighten this group of women and the public at large.

Keywords: ectopic pregnancy, risk factors, fertilized ovum, fallopian tube, reproductive accomplishments

\section{Introduction}

Ectopic pregnancy (EP) is an abnormal pregnancy in which a fertilized egg is implanted outside the uterine cavity. At least $90 \%$ of all EPs are located in the fallopian tube, and $80 \%$ of these are located in the ampullary segment of the tube. ${ }^{1-4}$ EP occurs in $1-2 \%$ of pregnancies and is associated with significant morbidity and mortality. Bleeding from ruptured fallopian tubes resulting from EP remains the most common cause of maternal mortality in the first trimester of pregnancy, ${ }^{5,6}$ and any pregnant woman could have an EP. ${ }^{2}$ However, pelvic inflammatory disease is the most common risk factor for EP. ${ }^{7}$ Other risk factors include the use of progesterone-only pills, intrauterine contraceptive devices (IUDs), endometriosis, previous tubal surgery, infertility, previous abortion, and assisted reproductive technology. The diagnosis is made primarily via transvaginal ultrasound (TVS) and supported by quantitative serum human chorionic gonadotropin (hCG). Any woman of reproductive age experiencing abnormal vaginal bleeding with or without abdominal pain is at risk of EP and should be 
followed closely until a diagnosis is made. It is now widely accepted that in the presence of hCG levels above the discriminatory zone of 1500-2500 IU/L, a normal intrauterine pregnancy (IUP), defined by the existence of a gestational sac, should be visible by TVS. Therefore, the absence of an intrauterine gestational sac when the hCG concentration is above the discriminatory zone implies an abnormal gestation. ${ }^{2}$ As a result of the limited resolution of TVS, not all EPs can be recognized, causing an "unknown location of pregnancy" result after an ultrasound. ${ }^{8}$ The patient's clinical condition, geographical location, and desire to reproduce and the available facilities all affect the treatment of EPs. Medical management is a safe and effective option in most clinically stable patients. Patients who have failed medical management, or are ineligible for medical management, or present with a ruptured EP or a heterotopic pregnancy are most often managed with excision via laparoscopy or, less commonly, laparotomy. ${ }^{9}$ Areas of active research include decreased time to diagnose EP, risk stratification in women with initial symptoms, and improved time to resolution via medical management. Therefore, it is of great significance to conduct statistical analyses on the disease characteristics of EP from large quantities of clinical data. This paper discusses the prevalence, associated risk factors, manifestations, and management of EP in the First Hospital of the Jilin University.

\section{Materials and Methods Subject}

This retrospective review included patients with EP who were treated at the First Hospital of the Jilin University between January 1, 2010 and December 31, 2014. The medical records of all participants were retrieved from the gynecology department, and during the review process, the confidentiality of patient data was ensured. The study was conducted in accordance with the Declaration of Helsinki (revised in 2013) and was approved by the Ethics Committee of the First Hospital of the Jilin University. All subjects signed informed consent.

\section{Inclusion and Exclusion Criteria}

Inclusion criteria: (1) diagnosis of EP, (2) >14 years old, and

(3) signed the informed consent. Exclusion criteria: (1) refusal to be hospitalized or (2) incomplete patient data.

\section{Research Methods}

According to the clinical protocol, women with pelvic pain and irregular vaginal bleeding following menstrual abnormalities were evaluated. In the absence of an intrauterine fetal sac, a tubal EP was diagnosed when $\beta$-hCG did not increase at the expected rate of $66 \% / 48$ hours expected of intrauterine pregnancies and a complex or solid adnexal mass was found on ultrasound, and a progesterone level $<25 \mathrm{ng} / \mathrm{mL}$ was used to support the diagnosis of an abnormal pregnancy. Women with stable hemodynamics received prospective therapy or methotrexate therapy, typically a single-dose regimen, and a single dose of $50 \mathrm{mg} / \mathrm{m}^{2}$.

\section{Main Observation Indexes}

Primary factors included age, gestational age, marital status, clinical presentation, diagnostic tools used, treatment options, intraoperative survey results, and associated risk factors.

\section{Statistical Analysis}

Statistical analysis was performed using the SPSS 22.0 software program (IBM, Chicago, USA). Continuous variables with a normal distribution were expressed as mean \pm standard deviation, continuous variables with a nonnormal distribution as median (quaternion range [IQR]), and categorical variables as frequency (percentage [\%]). For multiple comparisons on continuous data, each value was compared using a one-way method when the data conformed to a normal distribution, and non-parametric tests were used to compare non-normally distributed. Continuous data was tested using the chi-square test. $\mathrm{P}<$ 0.05 was considered statistically significant.

\section{Results}

\section{General Characteristics}

Of the 16,050 gynecological admissions during the fiveyear period under study, 1273 EPs were observed, with a prevalence rate of $7.93 \%$ of all gynecological admissions. The mean age of the study subjects was $30.62 \pm$ $6.19 \mathrm{y}$, and the incidence of EP in patients aged $<20$ or $>40$ y was extremely low (see Table 1).

\section{The Risk Factors}

In 156 cases $(12.3 \%)$, no risk factors were identified, but in the majority of cases, more than one risk factor was identified (see Table 2). 
Table I The Age Distribution

\begin{tabular}{|l|l|}
\hline Age (Years) & Number of Patients (\%) \\
\hline $15-19$ & $28(2.2)$ \\
$20-24$ & $187(14.7)$ \\
$25-29$ & $364(28.6)$ \\
$30-34$ & $344(27.0)$ \\
$35-39$ & $235(18.5)$ \\
$40-44$ & $102(8.0)$ \\
$45-52$ & $13(1.0)$ \\
\hline
\end{tabular}

Table 2 The Risk Factors Detected

\begin{tabular}{|l|l|l|}
\hline No. & Risk Factor & N (\%) \\
\hline 1 & History of PID & $342(26.9)$ \\
2 & IUD & $155(12.2)$ \\
3 & Irregular cycles & $127(10)$ \\
4 & Smoking & $29(2.3)$ \\
5 & Dysmenorrhea & $288(22.6)$ \\
6 & History of abortion & $777(61)$ \\
7 & History of pelvic surgery & $483(37.9)$ \\
8 & History of uterine cavity surgery & $491(38.6)$ \\
\hline
\end{tabular}

Table 3 First Symptoms

\begin{tabular}{|l|l|l|}
\hline No. & First Symptoms & N (\%) \\
\hline I & Bleeding & $101(7.9)$ \\
2 & Abdominal pain & $197(15.5)$ \\
3 & Asymptomatic & $42(3.3)$ \\
4 & Bleeding accompanied by abdominal pain & $830(65.2)$ \\
5 & Shock & $83(6.5)$ \\
6 & Digestive tract symptom & $20(1.6)$ \\
\hline
\end{tabular}

\section{Presentation}

As shown in Table 3, the first symptoms included bleeding, abdominal pain, bleeding accompanied by abdominal pain, shock, and digestive tract symptoms, but some patients were asymptomatic. The majority of patients $(1027,80.7 \%)$ presented with abdominal pain and bleeding, was elicited $(931,73.1 \%)$. A significant number of patients $(1220,95.8 \%)$ had a history of amenorrhea, and $42(3.3 \%)$ patients were asymptomatic (see Tables 2 and 3$)$.

\section{Site of EP}

A total of 929 patients had a tubal EP (73\%), and other sites included the ovary $(29,2.3 \%)$ and abdomen (7, $0.5 \%)$. It is noteworthy that 308 patients $(24.2 \%)$ had no definite EP site, including 299 patients who received medical and expectant treatment and 9 patients who underwent surgery.

The tubal EPs of 720, 64, and 145 patients occurred in the ampullary $(77.5 \%)$, cornual $(6.9 \%)$, and isthmic (15.6\%) regions, respectively.

\section{Diagnosis}

All patients were diagnosed via TVS and quantitative serum hCG (see Table 4). Three patients had normal levels and 1270 had elevated levels (19.80-318,000.00 IU/L; see Table 4).

\section{Treatment}

Patients with EP received surgical (974, 76.5\%), medical (223, 17.5\%), or expectant $(76,6 \%)$ management, and emergency laparotomy with unilateral salpingectomy was the most common surgical intervention. No mortality was recorded over the five years. There was no difference in serum hCG before treatment between the medical and expectant treatment groups, but the serum hCG was significantly higher in the surgical treatment group (see Table 5).

\section{Clinical Features of Patients without Previous Pregnancy or Delivery}

A total of 261 patients had no previous history of pregnancy, and the EP occurred in their first pregnancy. When compared with the patients with a history of pregnancy, there was no difference in the location of the EP, history of pelvic inflammation, number of days from when menstruation stopped, first symptoms, treatment plan, or blood hCG

Table 4 Trans-Vaginal Ultrasound Findings

\begin{tabular}{|l|l|l|}
\hline No. & Findings & N (\%) \\
\hline 1 & Pseudosac & $75(5.9)$ \\
2 & Live extrauterine fetus & $309(24.3)$ \\
3 & Isolated POD fluid & $583(45.8)$ \\
4 & Complex adnexal mass & $968(76.0)$ \\
5 & Simple ov cyst & $31(2.4)$ \\
\hline
\end{tabular}

Table 5 Level of Serum HCG (IU/L)

\begin{tabular}{|l|l|l|l|l|l|}
\hline $\begin{array}{l}\text { Treatment } \\
\text { Group }\end{array}$ & $\mathbf{N}$ & Min & Max & Median & $\mathbf{P}$ \\
\hline Expectant & 76 & 32.54 & $11,962.00$ & 361.40 & 0.982 \\
Medical & 223 & 30.40 & $19,800.00$ & 387.00 & 0.982 \\
Surgical & 974 & 3.62 & $318,000.00$ & 1534.75 & $<0.05$ \\
\hline
\end{tabular}


level before treatment. A total of 549 (43.1\%) patients were nulliparous. When compared with patients with a delivery history, there was no difference in the location of the EP, history of pelvic inflammation, number of days from when menstruation stopped, first symptoms, treatment plan, or blood hCG level before treatment. However, the percentage of patients with a history of pelvic surgery $(21.60 \%)$ was significantly lower in the nulliparous patients than in those with a history of delivery $(P<0.05)$.

\section{Discussion}

The prevalence of EP was $7.93 \%$ among all gynecological admissions. The majority of these patients were aged 25$34 \mathrm{y}$ and had a past history of abortion $(61 \%)$ and uterine cavity surgery $(38.6 \%)$, and a significant number were nulliparous (549, 43.1\%). Bleeding accompanied by abdominal pain were the most common presenting complaints (65.2\%). Unilateral salpingectomy was performed for most of these patients.

In recent years, with the prevalence of early sexual behavior and late childbearing among young people, there has been a lack of correct guidance on healthy reproduction, and the incidence of EP has increased year by year. ${ }^{10,11}$ The present study revealed that a significant proportion of nulliparous women have an EP. Hence, the follow-up fertility problems facing these patients are worthy of attention.

Women with an EP often have no identifiable factors. In the present study, 39\% of nulliparous patients had a history of abortion, accounting for a greater proportion than those with a history of pelvic inflammatory disease. ${ }^{10-18}$ When compared with women who have previously given birth, nulliparous women less often choose IUDs and oral contraceptives as contraceptive measures, and most of them use condoms, emergency contraceptives, and other measures. This increases the risk of contraceptive failure in these women. ${ }^{19}$ The incidence of abortion as a remedy for contraceptive failure is also relatively high in nulliparous women. Chronic infection may occur after an abortion or drug-induced abortion, or inflammatory infection may occur due to excessive vaginal bleeding and residual embryonic tissue, which may lead to tubal peristalsis disorder or lumen obstruction and could increase the risk of an EP.

EP has a wide range of clinical manifestations in women, ranging from being asymptomatic to experiencing severe circulatory failure. Unilateral abdominal pain with vaginal bleeding is often indicative of EP. Therefore, investigation via TVS is required for women of all reproductive ages with risk factors for EP. Non-gynecological symptoms, such as diarrhea, vomiting, or dizziness, may be most noticeable, but may not initially lead to a pregnancy test being conducted at the first evaluation, which may cause women to seek other healthcare services, such as medical or general surgical services.

In modern practice, the diagnosis of EP is based on the positive visualization of an additional uterine mass on $\mathrm{TVS}^{20}$ rather than the absence of an intrauterine gestational sac. At the first consultation, TVS had a sensitivity of $73.9-74.7 \%$ for detecting EPs, and in subsequent consultations, the overall sensitivity of TVS was $87-99 \%$ and the specificity was $94-99 \% .^{21,22}$ It is also important not to underestimate the psychological trauma associated with EP and the impact on future fertility.

When possible, an endoscopic approach is the desired route of access for ectopic pregnancies. There have clear advantages of the minimally invasive approach of laparoscopy for tubal pregnancies. These advantages include decreased operative time, a lower risk of postoperative adhesions, shorter inpatient stays, and an accelerated shorter return to activities of daily life as well as work. In this study, some patients with more abdominal bleeding and unstable vital signs underwent laparotomy. Most tubal ectopic pregnancies are located in the ampullary portion of the fallopian tube. For ectopic pregnancies located in the isthmic section of the tube, a partial or complete salpingectomy is recommended on the basis of concerns about the trophoblast infiltrating the tubal wall, with the risk of significant bleeding and low postoperative patency rates with conservative surgery. ${ }^{23}$

\section{Limitations}

This was a single-center trial, the sample size was small, and the clinical follow-up was short. Therefore, future studies are required to observe the clinical long-term prognosis of patients who have experienced an EP.

\section{Conclusion}

Over the past five years, EP has resulted in notable morbidity. A previous history of abortion and uterine cavity surgery were identified as the associated risk factors that limited the future reproductive potential of nulliparous women. Therefore, targeted health education campaigns should be created to enlighten this group of women and the public at large. 


\section{Ethics Approval and Consent to Participate}

The study was conducted in accordance with the Declaration of Helsinki (as was revised in 2013). The study was approved by Ethics Committee of the First Hospital of Jilin University. All subjects signed informed consent, and a legal guardian of patients under 18 years of age provided informed consent.

\section{Acknowledgments}

We are particularly grateful to all the people who have given us help on our article.

\section{Funding}

There is no funding to report.

\section{Disclosure}

The authors declare that they have no competing interests.

\section{References}

1. Escobarpadilla B, PerezLópez CA, MartínezPuon H. [Risk factors and clinical features of ectopic pregnancy]. Rev Med Inst Mex Seguro Soc. 2017;55:278-285. Swedish.

2. ASRM. The practice committee of the American Society for Reproductive Medicine. Medical treatment of ectopic pregnancy: a committee opinion. Fertil Steril. 2013;100(3):638-644. doi:10. 1016/j.fertnstert.2013.06.013

3. Hamid AAA, Yousry A, Radi SAE, et al. Clinical audit of ectopic pregnancy. Electr Phys. 2017;9:4009-4013. doi:10.19082/4009

4. Shaw JL, Diamandis EP, Horne AW, Barnhart K, Bourne T, Messinis IE. Ectopic pregnancy. Clin Chem. 2012;58:1278-1285. doi:10.1373/clinchem.2012.184168

5. Chan JW. Acute bilateral simultaneous PION after ectopic pregnancy-related haemorrhage. Eye. 2006;20:747-748. doi:10.1038/ sj.eye. 6702010

6. Khan KS, Wojdyla D, Say L, Gulmezoglu AM, Van Look PF. WHO analysis of causes of maternal death: a systematic review. Lancet. 2006;367:1066-1074. doi:10.1016/S0140-6736(06)68397-9

7. Goller JL, Livera AMD, Rebecca JG, et al. Rates of pelvic inflammatory disease and ectopic pregnancy in Australia, 2009-2014: ecological analysis of hospital data. Sex Transm Infect. 2018;94:534-541. doi:10.1136/sextrans-2017-053423

8. Young L, Barnard C, Lewis E, et al. The diagnostic performance of ultrasound in the detection of ectopic pregnancy. $N Z$ Med $J$. 2017;130:17-22.

International Journal of General Medicine

\section{Publish your work in this journal}

The International Journal of General Medicine is an international, peer-reviewed open-access journal that focuses on general and internal medicine, pathogenesis, epidemiology, diagnosis, monitoring and treatment protocols. The journal is characterized by the rapid reporting of reviews, original research and clinical studies
9. Panelli DM, Phillips CH, Brady PC. Incidence, diagnosis and management of tubal and nontubal ectopic pregnancies: a review. Fertil Res Pract. 2015;15(1):15.

10. Belics Z, Gerecz B, Csakany MG. Early diagnosis of ectopic pregnancy. Orv Hetil. 2014;155:1158-1166. doi:10.1556/OH.2014. 29933

11. Mummert T, Gnugnoli DM. Ectopic Pregnancy. In: StatPearls. Treasure Island (FL): StatPearls Publishing; August 11, 2021.

12. Tsikouras P, von Tempelhoff GF, Rath W. [Epidemiology, risk factors and risk stratification of venous thromboembolism in pregnancy and the puerperium]. Z Geburtshilfe Neonatol. 2017;221:161-174. German. doi:10.1055/s-0043-107618

13. Miller EC, Gatollari HJ, Too G, et al. Risk factors for pregnancy-associated stroke in women with preeclampsia. Stroke. 2017;48:1752. doi:10.1161/STROKEAHA.117.017374

14. Tao G, Patel C, Hoover KW. Updated estimates of ectopic pregnancy among commercially and Medicaid-insured women in the United States, 2002-2013. South Med J. 2017;110:18-24. doi:10.14423/ SMJ.0000000000000594

15. Suzuki S, Hiraizumi Y, Satomi M. History of abortion and perinatal outcomes associated with preeclampsia in nulliparous Japanese women. J Mater Fetal Neonatal Med. 2010;23:1318-1319. doi:10. 3109/14767050903551517

16. Rose SB, Garrett SM. Postabortion initiation of long-acting reversible contraception by adolescent and nulliparous women in New Zealand. $J$ Adolescent Health. 2016;58:160-166. doi:10.1016/j.jadohealth. 2015.09.025

17. Behery MME, Siam S, Seksaka MA. Reproductive performance in the next pregnancy for nulliparous women with history of first trimester spontaneous abortion. Arch Gynecol Obstet. 2013;28 8:939-944. doi:10.1007/s00404-013-2809-9

18. Brown J, Sciscione A, Hoffman M, et al. The impact of prior abortion on labor outcomes among nulliparous women. Contraception. 2014;90:302. doi:10.1016/j.contraception.2014.05.037

19. Hatcher RA, Warner DL. New condoms for men and women, diaphragms, cervical caps, and spermicides: overcoming barriers to barriers and spermicides. Curr Opin Obstet Gynecol. 1992;4: 513-521. doi:10.1097/00001703-199208000-00004

20. Johnson S, Bonello MR, Li Z, Hilder L, Sullivan EA Maternal deaths in Australia 2006-2010. Maternal deaths series no 4 Cat no PER 61 Canberra: AIHW; 2014.

21. Kirk E, Papageorghiou AT, Condous G, Tan L, Bora S, Bourne T. The diagnostic effectiveness of an initial transvaginal scan in detecting ectopic pregnancy. Hum Reprod. 2007;22:2824-2828. doi:10.1093/ humrep/dem 283

22. Lattouf I, Lu C, Pixton S, Reid S, Condous G. Is there a difference in the behaviour and subsequent management of ectopic pregnancies seen at first scan compared to those ectopic pregnancies which commence as pregnancies of unknown location? Aust N Z J Obstet Gynaecol. 2016;56:107-112. doi:10.1111/ajo.12434

23. Ozcan MCH, Wilson JR, Frishman GN. A systematic review and meta-analysis of surgical treatment of ectopic pregnancy with salpingectomy versus salpingostomy. J Minim Invasive Gynecol. 2021;28 (3):656-667. doi:10.1016/j.jmig.2020.10.014

across all disease areas. The manuscript management system is completely online and includes a very quick and fair peer-review system, which is all easy to use. Visit http://www.dovepress.com/ testimonials.php to read real quotes from published authors. 